\title{
Enfermeras y practicantes durante la epidemia de gripe de 1918: Análisis a través de la prensa española
}

Nurses and practitioners during the flu epidemic of 1918: Analysis through the Spanish press Enfermeiras e profissionais durante a epidemia de gripe de 1918: Análise através da imprensa española

Laura Almudéver Campo ${ }^{1}$, Ramón Camaño Puig²

\author{
${ }^{1}$ Doctora en Enfermería por la Universidad de Valencia (2016). Profesora asociada. Departamento de \\ Enfermería. Universidad de Valencia. Enfermera en Centro de Salud Alfahuir (Valencia) \\ 2 Doctor Europeo por Leeds Metropolitan University (1997). Profesor titular. Departamento de Enfermería. \\ Universidad de Valencia \\ Cómo citar este artículo en edición digital: Almudéver Campo, L. E Camaño Puig, R. (2018). \\ Enfermeras y practicantes durante la epidemia de gripe de 1918: Análisis a través de la prensa española. \\ Cultura de los Cuidados (Edición digital), 22(52). Recuperado de \\ http://dx.doi.org/10.14198/cuid.2018.52.10 \\ Correspondencia: Laura Almudéver Campo. C/ Saler, n.ํ44. C. P. 46290 Alcàsser (València) \\ Correo electrónico: Laura.Almudever@uv.es \\ Recibido: 22/03/2018; Aceptado: 14/05/2018
}

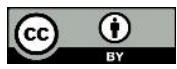

\section{ABSTRACT}

Objective: The influenza epidemic of 1918 is considered one of the most lethal in the history of mankind, causing a high morbidity and mortality that was reflected in the Spanish press. Therefore, we intend to address the epidemic-related events in relation to nursing professionals from a journalistic perspective.

Material and method: From ten Spanish newspaper heads, the information on nursing professionals has been extracted, from January 1, 1918 to December 31, 1920, proceeding to its thematic organization and characterization.

Results: A total of 555 units of analysis were obtained, which addressed aspects of recognition, creation of professional profiles, beginning of academic course, as well as wage demands, strikes and care that were offered.

Conclusion: We could say that the number of information is scarce, which raises an invisibility of nursing professionals, which is extended to the present.

Keywords: Flu epidemic, flu 1918, nursing professionals.

\section{RESUMO}

Objetivo: A epidemia de gripe de 1918 é considerada uma das mais letal da história da humanidade, causando alta morbidade e mortalidade que se refletiu na imprensa espanhola. Portanto, pretendemos abordar os eventos relacionados à epidemia em relação aos profissionais de enfermagem sob a perspectiva jornalística. 
Material e método: De dez cabeças de jornais espanhóis foram extraídas informações sobre profissionais de enfermagem, de 1. de janeiro de 1918 a 31 de dezembro de 1920, procedendo à sua organização e caracterização temática.

Resultados: Foram obtidos 555 unidades de análise, que abordaram aspectos de reconhecimento, criação de perfis profissionais, início do curso, bem como reivindicações salariais, greves e cuidados oferecidos.

Conclusão: Poderíamos dizer que o número de informações é escasso, o que sugere uma invisibilidade dos profissionais de enfermagem, que se estende ao presente.

Palavras-chave: Epidemia de gripe, gripe de 1918, enfermagem, estagiários.

\section{RESUMEN}

Objetivo: La epidemia de gripe de 1918 se considera una de las más letales de la historia de la humanidad, ocasionando una alta morbilidad y mortalidad que quedó reflejada en la prensa española. Por ello, pretendemos abordar los acontecimientos relativos a la epidemia en relación con los profesionales de Enfermería desde la perspectiva periodística.

Material y método: A partir de diez cabeceras de periódicos españoles se ha procedido a la extracción de las informaciones relativas a profesionales de Enfermería, desde el 1 de enero de 1918 hasta el 31 de diciembre de 1920, procediendo a su organización $\mathrm{y}$ caracterización temática.

Resultados: Se obtuvieron un total de 555 unidades de análisis, que abordaron aspectos de reconocimiento, creación de perfiles profesionales, comienzos de curso, así como reivindicaciones salariales, huelgas y cuidados que se ofrecían.
Conclusión: Podríamos decir que el número de informaciones es escaso, lo que plantea una invisibilidad de los profesionales de enfermería, que se extiende hasta la actualidad.

Palabras clave: Epidemia de gripe, gripe de 1918, enfermería, practicantes

\section{INTRODUCCIÓN}

A lo largo del siglo XIX y principios del XX convivieron en España diferentes profesiones $y$ oficios sanitarios con competencias y marcos legislativos distintos caracterizados por orientarse al ejercicio de la actividad siempre bajo la dirección del médico (Herrera, 2000; Camaño, 2008). En esta línea, la epidemia de gripe de 1918 constituyó un evento que puso a prueba las estructuras organizativas y profesionales en el ámbito de la salud, ya que es considerada una de las más letales de la historia de la humanidad, ocasionando una alta morbilidad y mortalidad que quedó reflejada en la prensa española.

Podríamos decir que en nuestro país, los ancestros profesionales de la actual enfermería existentes en ese momento fueron los practicantes que habían sido creados mediante la Ley Moyano de 1857, creándose la primera Escuela de enfermería en Madrid, por Rubio y Galí, en el Instituto Operatorio y denominada Santa Isabel de Hungría, a la que siguieron otras fundadas por diferentes organizaciones a lo largo del país.

Mediante la Real Orden de 6 de julio de 1864 la Reina Isabel II confirió a la Cruz Roja el carácter oficial, y su nuera, la reina María Cristina de Habsburgo, ayudó de una manera decisiva a la Institución, amparando, estimulando y propiciando la reorganización de la Cruz Roja Española. 
Pero no fue hasta el 28 febrero de 1917 cuando se dictó un Real Decreto por el cual se aprobaban las instrucciones generales para la organización y constitución del Cuerpo de Damas Enfermeras de la Cruz Roja Española.

En esos años, la Enfermería en España se encontraba en la búsqueda de su identidad profesional, pues su formación estaba dirigida por médicos, los cuales adiestraban a las enfermeras y los practicantes para ser eficientes y sumisos ayudantes y cuya formación en el caso de las enfermeras, iba acompañada de la tutela religiosa (Herrera, 1996; Feria, León, Macías, Barquero, Marquínez y Cárdenas, 2004; Parrilla y García, 2004; Ortiz, 2005).

La enfermería fue reconocida oficialmente mediante la Real Orden de 7 de mayo de 1915 que autorizaba para ejercer la enfermería a aquellas personas que probaran tener el conocimiento necesario ya pertenecieran o no a una orden religiosa. Dicho decreto causó un conflicto con los practicantes, que mostraron su desacuerdo e insatisfacción a través de manifestaciones, alegando que la existencia de enfermeras contribuiría a la división de las Clases de Auxiliar Sanitario. El título de enfermera se reconoció como una profesión distinta a la de los practicantes y se estableció el primer programa oficial para su formación según las directrices de la Ley de Instrucción Pública de 1904 (Domínguez, 1986; Herrera, 1996; Santo Tomás, 1997; Parrilla y García, 2004; Ortiz, 2005).

Dicho grupo profesional femenino, bajo la nueva denominación de Enfermería, tendría orientados los contenidos de sus estudios a la obtención de conocimientos y habilidades que permitiesen llevar a cabo tareas de atención a enfermos, los cuidados del paciente, la realización de técnicas así como la asistencia social y de colaboración sanitaria (Del Puerto, 2011).

Los practicantes, por otra parte, eran considerados herederos de los antiguos cirujanos ministrantes, por lo que sus cometidos estuvieron relacionados con los aspectos técnicos y de la cirugía menor, encargándose de la vigilancia y custodia de los enfermos. Entre sus funciones destacaban el auxiliar a los médicos en las operaciones, asistir en las autopsias, practicar las curaciones de los enfermos, administrar las medicaciones y llenar los recetarios. Aspectos que han condicionado, posiblemente, el rol de los profesionales de Enfermería en los últimos años.

Esta investigación tiene como objetivo el estudio y análisis, a través de la prensa española, de la labor de los profesionales de Enfermería durante la epidemia de gripe de 1918, enfatizando en los cuidados que se aplicaban a los enfermos durante el brote epidémico y quiénes dispensaban dichos cuidados.

\section{MATERIAL Y MÉTODO}

Para llevar a cabo dicho estudio, se ha realizado un análisis de la prensa española desde el 1 de enero de 1918 al 31 de diciembre de 1920. En primer lugar, se seleccionaron las cabeceras incluidas en el catálogo de la Hemeroteca Digital de la Biblioteca Nacional, que se editaran de manera continuada a lo largo de los tres años de nuestro estudio y cuyo número de ejemplares anuales fuera superior a 100, en términos de obtener un número suficiente de ejemplares. No siendo la elección de este número arbitraria, dado que muchas de las cabeceras del momento tuvieron una vida efímera o un bajo nivel de continuidad.

Se obtuvieron 8 cabeceras a las que añadimos los periódicos $A B C$ y $L a$ Vanguardia, que no estaban incluidos en el 
catálogo de la Hemeroteca Digital de la Biblioteca Nacional y que consideramos de gran relevancia en el periodo de tiempo estudiado. La selección final quedó compuesta por: $A B C, L a$ Correspondencia de España (LCE), El Heraldo de Madrid, El Imparcial, El País, El Sol, La Acción, La Época, El Globo y La Vanguardia,

Posteriormente se realizó una búsqueda en los mismos sobre los conceptos 'enfermera', 'practicante' y 'enfermero', y se seleccionaron todas las publicaciones que incorporaron referencias a dichos conceptos, en el periodo de 1918-1920, a excepción de los anuncios publicitarios. Con las unidades de análisis obtenidas, se procedió a su estudio para eliminar aquellas unidades que contenían a la vez dos o más términos estudiados, con el fin de eliminar las duplicidades. A continuación, se realizó un análisis cuantitativo y una descripción temática, estableciendo categorías $\mathrm{y}$ extractando de cada una de las unidades de análisis la correspondiente referencia a los conceptos de interés en el trabajo.

\section{RESULTADOS}

La búsqueda de los conceptos 'enfermera', 'practicante' y 'enfermero' ha generado un total de 555 unidades de análisis, tal y como se puede apreciar en la Tabla 1, que muestra el número de unidades de análisis distribuidas según el término buscado, el año elegido y el periódico seleccionado.

TABLA 1: Unidades de análisis según periódico, término y año

\begin{tabular}{|c|c|c|c|c|c|c|c|c|c|c|}
\hline \multirow[b]{2}{*}{ Cabecera } & \multicolumn{3}{|c|}{ Enfermera } & \multicolumn{3}{|c|}{ Practicante } & \multicolumn{3}{|c|}{ Enfermero } & \multirow[b]{2}{*}{ Total } \\
\hline & 1918 & 1919 & 1920 & 1918 & 1919 & 1920 & 1918 & 1919 & 1920 & \\
\hline$A B C$ & 7 & 5 & 9 & 3 & 10 & 9 & 6 & 2 & 2 & 53 \\
\hline El Globo & 2 & 2 & 2 & 3 & 7 & 5 & 0 & 1 & 1 & 23 \\
\hline El Heraldo & 8 & 2 & 13 & 1 & 1 & 1 & 1 & 1 & 2 & 30 \\
\hline El Imparcial & 4 & 4 & 3 & 8 & 5 & 11 & 3 & 2 & 2 & 42 \\
\hline El País & 0 & 0 & 0 & 3 & 2 & 3 & 0 & 0 & 0 & 8 \\
\hline El Sol & 6 & 8 & 5 & 14 & 6 & 5 & 2 & 6 & 4 & 56 \\
\hline La Acción & 12 & 4 & 2 & 12 & 12 & 14 & 0 & 4 & 2 & 62 \\
\hline La Época & 5 & 9 & 5 & 3 & 3 & 7 & 1 & 0 & 0 & 33 \\
\hline La Vanguardia & 36 & 32 & 50 & 10 & 3 & 5 & 10 & 7 & 3 & 156 \\
\hline$L C E$ & 6 & 7 & 32 & 12 & 13 & 13 & 1 & 3 & 5 & 92 \\
\hline $\begin{array}{l}\text { Totales } \\
\text { parciales }\end{array}$ & 86 & 73 & 121 & 69 & 62 & 73 & 24 & 26 & 21 & 555 \\
\hline Totales & & & 280 & & & 204 & & & 71 & 555 \\
\hline
\end{tabular}

Una vez recogida y tratada la información cuantitativa, se realizó un cruce de los vocablos para analizar el número de unidades de análisis que contenían a la vez varios de los términos estudiados, encontrando 5 coincidencias, concretamente en los términos: Enfermera + Practicante: $E l$ Sol (29-10-1918:8); Enfermera + Enfermero: La Vanguardia (27-1-1918:18 / 29-9-1919:10); y Practicante + Enfermero: ABC (20-9-1919:14) 
y La Vanguardia (19-10-1919:20). Por tanto, tras restar las coincidencias, obtuvimos un total de 550 unidades.

Como se puede observar en la Tabla 1, el término enfermera fue del que más unidades de análisis se publicaron, concretamente 280, seguido del término practicante con 204, y del término enfermero con 71. Durante el año 1920 tuvo lugar la publicación del mayor número de unidades de análisis con el término enfermera, obteniendo 121 unidades, seguido del año 1918 con 86 y del año 1919 con 73. Respecto a los periódicos, oscilaron en número de publicaciones desde La Vanguardia, que fue el medio que más veces publicó el término enfermera con 118 unidades, a $E l$ Globo que solo lo publicó en 6 ocasiones, o incluso $E l$ País, que no utilizaron tal término.

En dichas unidades de análisis, el concepto enfermera ha aparecido muy ligado a la institución de la Cruz Roja, una institución que tuvo un destacado papel en el desarrollo de la Enfermería de nuestro país. En este sentido, en La Vanguardia (1-5-1918:12) se recogía el fin de curso de las damas enfermeras de la Cruz Roja en Valladolid, las cuales marchaban a Madrid para que les hicieran entrega del título de enfermeras por la propia Reina. Posteriormente desde Tortosa en el mismo medio (31-5-1918:13) se plasmaba la inauguración de las clases del curso teórico de damas enfermeras de la Cruz Roja de esa ciudad. Desde $A B C$ (8-61918:18) y La Acción (8-6-1918:4) se decía que se iba a hacer entrega de las medallas, brazales y diplomas a las nuevas damas enfermeras de la Cruz Roja. En $A B C$ (7-111918:26) se informaba de la apertura de la matrícula oficial para los estudios teóricoprácticos de damas enfermeras de la Cruz Roja. Por su parte, en El Sol (19-12-1918:10) y dado que se recogían noticias de la Gran Guerra (1914-1918) en curso, se indicaba que las enfermeras francesas y damas de la Cruz Roja prestaban servicio en todos los hospitales de evacuación; y en La Vanguardia (28-12-1918:13) se plasmaba la inauguración en Trieste de la primera Escuela de enfermeras, con destino a prestar servicio en las ambulancias de la Cruz Roja italiana.

Medios como El Sol (10-4-1919:2), La Época (11-4-1919:4), La Correspondencia de España (2-6-1920:12), $\quad A B C \quad(29-6-1920: 16) \quad$ y $\quad L a$ Vanguardia (6-1-1919:7, 29-4-1919:12, 10-91919:11, 29-2-1920:14, 4-4-1920:4 y 30-61920:8), realizaron diversas publicaciones sobre los cuidados y la labor de las Damas enfermeras de la Cruz Roja. Por otra parte, los cuidados ofrecidos por las enfermeras tituladas también quedaron reflejados en la prensa de la época, así quedó demostrado en El Sol (17-1-1918:8), El Imparcial (8-2-1918:2), La Acción (16-3-1918:5, 28-4-1918:2 y 14-101918:3), El Heraldo (17-3-1919:1), ABC (19-21920:13), La Época (10-6-1920:3) y La Correspondencia de España (27-7-1920:12 y 159-1920:2), que manifestaron la gran tarea que realizaban las enfermeras, convirtiéndose en gran ayuda de los cirujanos.

Así mismo, era habitual encontrar en los medios estudiados publicaciones en referencia a los fallecimientos y entierros de enfermeras que habían sucumbido a causa de la gripe: La Época (12-4-1918:2), El Heraldo de Madrid (30-5-1918:4), La Vanguardia (12-61918:10), El Sol (29-10-1918:3) y El Imparcial (11-9-1919:4). Como consecuencia de tales padecimientos y en su caso fallecimientos, se produjo una disminución de las enfermeras en los distintos hospitales españoles, apareciendo varias notas en referencia al escaso número de enfermeras. Por ejemplo, en El Sol (18-1-1920:4 y 3-2-1920:12) se decía que en los hospitales extranjeros había una enfermera para cada cinco enfermos, mientras que aquí había una para cada cien. Según parece, la escasez de enfermeras no 
era circunstancial sino endémica, situación que podría haberse hecho extensiva hasta nuestros días.

También encontramos noticias acerca de dos monumentos dedicados a enfermeras, el de la Reina Isabel levantado en Bruselas y recogido en $A B C$ (1-9-1919:11), La Época (1-91919:4) y La Vanguardia (1-9-1919:8); y el de la enfermera inglesa Edith Cavell, que atendió y cuidó a los heridos de la Primera Guerra Mundial, publicado en La Vanguardia (31-12-1918:12), La Correspondencia de España (10-5-1919:2 y 15-5-1919:6) y El Globo (15-11920:2).

Otro de los temas por los que se mencionó el concepto enfermera fue debido a la inclusión de esta categoría profesional entre los componentes de la tripulación de las embarcaciones: La Vanguardia (31-1-1918:11, 3-7-1918:11 y 20-6-1919:12) y El Heraldo de Madrid (23-11-1918:3). Asimismo, el periódico La Vanguardia recogía las diferentes conferencias dirigidas a las alumnas enfermeras e impartidas por médicos, sobre alimentación e higiene (10-11918:5, 16-3-1918:2 y 26-6-1919:3), poniéndose de manifiesto la dependencia que tenían las enfermeras de los médicos, situación que venía arrastrándose desde el siglo XIX, momento en que Florence Nightingale, se planteó la actuación de las enfermeras desde una perspectiva de total dependencia respecto a la profesión médica.

En referencia al término practicante durante el año 1920 se publicaron 73 unidades, siendo el año en el que más unidades de análisis aparecieron, seguido muy de cerca por el año 1918 con 69 y del año 1919 con 62. Los periódicos LCE y La Acción con 38 unidades cada uno, fueron los que más veces publicaron dicho término. El País con 8 unidades y $\mathrm{El} \mathrm{Heraldo} \mathrm{con} \mathrm{3,} \mathrm{son} \mathrm{las} \mathrm{cabeceras}$ que menos veces lo publicaron (Tabla 1).

En las unidades de análisis el concepto practicante ha estado relacionado con la persona adecuada para realizar la cura de heridas, tal y como lo muestran los extractos publicados por El Imparcial (17-2-1918:1), La Época (17-2-1918:2) y El Sol (1-4-1918:5). Más adelante, el 3 de junio de 1918, los periódicos $A B C$, La Acción y La Correspondencia de España se hacían eco de una información desde Granada, sobre la cura a un herido. Asimismo, en El País (14-8-1918:3) se recogía un suceso sobre una riña sangrienta, en que el practicante le apreció varias heridas a uno de los implicados. En 1919, La Correspondencia de España (24-7-1919:4), La Acción (15-8-1919:3) y El Sol (13-9-1919:3) publicaron informaciones requiriendo la actuación de practicantes. En 1920, El Imparcial (7-1-1920:1), El Globo (17-1-1920:2 y 9-7-1920:3), El País (6-5-1920:1 y 14-111920:3) y $A B C(22-5-1920: 5,15-7-1920: 17$ y 39-1920:20) también informaron sobre la cura de heridos. Finalmente, en una información aparecida en El Globo (17-1-1920:2) se mencionaba que un practicante le aplicó una inyección a un enfermo.

El empeoramiento de la salud y fallecimiento de los practicantes fue objeto de noticia con publicaciones en El Imparcial (25-9-1918:3 y 5-10-1918:4) y El Heraldo (2-121920:4); así como la falta de practicantes en algunos pueblos y ciudades debido al aumento de la morbilidad y mortalidad como consecuencia de la epidemia de gripe: La Correspondencia de España (27-10-1918:2, 311-1918:5 y 31-12-1918:2), La Acción (31-121918:2 y 2-1-1919:3) y El Sol (28-12-1919:4).

En dos notas periodísticas publicadas en La Acción (28-1-1919:4 y 29-1-1919:5) se plasmaban los acuerdos del III Congreso de Sanidad Civil; y en La Correspondencia de España (21-8-1919:5) se informaba de lo expuesto en la Quinta Asamblea Regional en Málaga, describiendo las relaciones que debían existir entre profesionales sanitarios, 
los practicantes, las matronas, las enfermeras y el personal médico. A los dos meses en El País (21-10-1919:2) se publicaban las bases de la práctica profesional para los practicantes, al igual que lo hacían al año siguiente El Globo (2-8-1920:3), La Correspondencia de España (21-9-1920:3) y La Vanguardia (30-10-1920:11). Una información que ponía de manifiesto el papel de dichos profesionales como meros auxiliares de los médicos, donde se indicaba que los practicantes eran colaboradores indispensables de los médicos y se sostenía que en todo servicio facultativo debía figurar un practicante como auxiliar inmediato del médico.

Los practicantes también formaban parte de la tripulación de los buques y de la Armada, exponiéndose diversas informaciones al respecto. En el periódico $L a$ Acción (6-2-1918:5), ABC (20-9-1919:14) y El Imparcial (20-9-1919:1) se mencionaba la tripulación de los buques y los ascensos e ingresos de nuevos practicantes. En cuanto a la tripulación de la Armada, se exponía información parecida en La Acción (23-71918:4, 17-9-1919:4 y 13-3-1920:5) y El Globo (17-10-1918:2 y 10-3-1919:2).

En los textos analizados también se informó de los sueldos de los practicantes, exponiéndose en el periódico El Sol (21-91918:3 y 22-9-1918:2), $A B C$ (8-9-1919:8) y El Globo (8-9-1919:1-3). Asimismo, varios periódicos informaron de la huelga que convocaron los practicantes: $A B C$ (29-121919:11, 25-8-1920:7 y 2-11-1920:9) y El Globo (31-12-1919:2).

Respecto del término enfermero se publicaron en el año 1919, 26 unidades de análisis, seguido de 24 en 1918 y de 21 en 1920. Respecto a la distribución por periódicos encontramos una amplia variabilidad, oscilando desde las 20 publicaciones de La Vanguardia, la cabecera que más veces publicó el término enfermero, a La Época que solo lo hizo en una ocasión, o El País, que no lo publicó.

A principios del siglo $\mathrm{XX}$ el trabajo de los enfermeros, una denominación atribuida a un personal sin titulación, que más tarde fue utilizada para referirse a los practicantes en el ámbito de la psiquiatría, consistía en realizar labores dentro del campo de actuación de auxiliares, celadores, vigilantes o personal de limpieza actuales. Algunas unidades de análisis en las que aparecía el concepto enfermero se referían a este cometido: ABC (18-2-1918:14, 20-10-1918:13 y 29-1-1919:8) y El Imparcial (18-2-1918:2 y 49-1918:5). En El Sol (10-6-1919:12, 4-5-1920:12 y 18-5-1920:16), se recogían tres informaciones acerca de los manicomios españoles, diciendo que los enfermeros hacían formar a los enfermos a lo largo de las paredes, que hacían abuso de los procedimientos de curación como la camisa de fuerza y que además, tenían a su cargo a veinte, treinta, cincuenta o más alienados. $Y$ en El Imparcial (4-9-1918:5) se indicaba que un médico le hizo una recomendación a un celador enfermero, que consistía en apretar el estómago a un enfermo hasta que echase el agua que había tragado y que guardaba dentro.

$\mathrm{Al}$ igual que el concepto enfermera $\mathrm{y}$ practicante, el término enfermero también se usó para hacer referencia a la tripulación de las embarcaciones, concretamente apareció una única información en varias cabeceras identificando a Florencio Pérez, un enfermero con su nombre y apellido en $L a$ Acción y La Correspondencia de España (19-91919:6), ABC (20-9-1919:14) y El Globo (22-91919:1).

En tres ejemplares, $A B C$ (24-6-1920:17 y 217-1920:14) y La Acción (21-7-1920:6) se publicó una noticia que informaba sobre la sustitución de los enfermeros de los 
hospitales de Melilla por hermanas de la Caridad, algo habitual en el momento que obedecía a criterios de expansión de la orden religiosa en nuestro país. También se informó de las diferentes huelgas que los enfermeros propusieron en distintas ocasiones: La Vanguardia (14-1-1919:13, 29-91919:10, 5-2-1920:6 y 8-2-1920:6). Asimismo, aparecieron dos informaciones sobre premios y retribuciones de los enfermeros: La Vanguardia (27-1-1918:18) y El Heraldo de Madrid (31-12-1919:3). Y por último, se hizo una única referencia a una conferencia que se iba a impartir para enfermeros, y que quedó plasmada en La Vanguardia (21-11919:5).

\section{DISCUSIÓN}

En este estudio se ha analizado la realidad socio-sanitaria del periodo de tiempo comprendido entre 1918 y 1920, situando los términos enfermera, practicante $\mathrm{y}$ enfermero en las rutinas cotidianas de la información, determinando el contexto en el que se ubica la investigación, para tratar de conocer la imagen de los profesionales de Enfermería en la prensa durante la epidemia de 1918.

A lo largo de nuestro trabajo hemos podido evidenciar una situación de mimetismo entre la mayoría de los periódicos estudiados, encontrando titulares e informaciones muy similares en ellos, en ocasiones, idénticos. Esto pudo deberse a que las informaciones eran enviadas a los periódicos por las agencias de noticias, sin que los redactores las modificaran ni ampliaran, publicándolas tal cual eran recibidas de la agencia, primando más la rapidez en su publicación que la calidad de las mismas. Se trataba de textos escritos en el mismo momento en que estaban ocurriendo los acontecimientos, incorporándose a los medios con inmediatez y espontaneidad. Además, la epidemia de gripe fue una temática publicada en portada en algunos de los ejemplares analizados, lo que indica la intención de los medios de darle un lugar privilegiado en la jerarquía de las noticias.

Basándonos en los datos obtenidos en el análisis de los periódicos, hemos observado que durante la epidemia de gripe de 1918 sólo se aplicaban cuidados básicos y domésticos para aliviar a los afectados del virus, que consistían principalmente en medidas higiénicas para evitar la propagación de la enfermedad. Dichos cuidados eran aplicados por las mujeres en las familias y las enfermeras en las instituciones sanitarias, que fueron quienes prestaron ayuda, cuidado y aseo a los enfermos, como respuesta a las necesidades básicas específicas de cada paciente. Por su parte, los practicantes, en su mayoría hombres, se encargaban de la asistencia auxiliar médico-quirúrgica domiciliaria y/o en consultas privadas, con especial presencia en el mundo rural donde apenas había médicos (Bernabeu, Carrillo, Galiana, García y Trescastro, 2013).

Por tanto, podemos afirmar que los profesionales de Enfermería jugaron un papel crucial en el cuidado de los enfermos, ya que, en ausencia de una infraestructura formal de salud pública, precariedad de los servicios sanitarios, y carente de medios terapéuticos, fueron el único remedio para intentar contener la epidemia, convirtiéndose en la primera línea de defensa contra la enfermedad. Sin embargo, un gran número de profesionales sanitarios estuvieron atacados por la gripe, provocando la carencia de los mismos y siendo insuficientes para la asistencia a los enfermos.

En este contexto cabe mencionar la figura 
de las Damas Enfermeras de la Cruz Roja, que prestaron asistencia como voluntarias ayudando a los enfermos de gripe, tal y como hemos visto en varias notas periodísticas. Por tanto, se puede afirmar que la prensa española colaboró con la marcha de la epidemia, facilitando la difusión de estrategias de captación de voluntarios sanitarios para suplir las deficiencias de personal, como lo fueron las damas enfermeras de la Cruz Roja. Sin embargo, no queda constancia de que esta situación favoreciera a los profesionales de Enfermería, ni si desarrollaron sus competencias y/o mejoraron su imagen social.

\section{CONCLUSIONES}

En general, se aprecia que los diferentes periódicos analizados prestaron atención a las enfermeras, los practicantes y los enfermeros desde diferentes puntos de vista: la realización de cursos, especialmente aquellos relativos a la Cruz Roja, una institución ligada a la Corona; el reconocimiento de este grupo profesional y algunas de sus figuras prominentes mediante monumentos; o la creación de perfiles novedosos como los practicantes, enfermeras y algún enfermero vinculados a las tripulaciones de buques y a la Armada. Siendo de destacar las unidades dedicadas a describir las tareas que componían el trabajo de las enfermeras, los practicantes y los enfermeros; en el caso de los practicantes haciendo una referencia clara a la administración de inyectables y curas. Todo ello, en términos de satisfacción $y$ promoción por parte de los diferentes grupos del rol de auxiliares del médico. Adicionalmente, en algunas unidades de análisis queda más o menos manifiesto el grado de conflictividad existente mediante la información sobre las huelgas y los sueldos.

Finalmente, los periódicos también se hicieron eco del nivel de afectación de los profesionales por la epidemia de gripe y en algunos casos, del fatal desenlace de los profesionales en cumplimiento de su labor. Por tanto, podríamos decir que el tratamiento de estas figuras fue escaso y condicionado por una época convulsiva y de crisis como fue la concurrencia de la I Guerra Mundial y la pandemia de gripe, pero que nos muestra, aunque sea mínimamente, una profesión generando cambios y ajustes en momentos conflictivos.

Como reflexión final, de hecho, podríamos decir que en conjunto hay una escasez de informaciones respecto de los profesionales de Enfermería, de los cuidados que ofrecían y las labores que realizaban, en términos cuantitativos, una situación que pudiera etiquetarse de invisibilidad y subordinación que ha perdurado a lo largo de todo el siglo XX y que en la actualidad todavía sigue vigente, lo que ha influenciado socialmente en el escaso reconocimiento de la profesión.

\section{BIBLIOGRAFÍA}

- Bernabeu, J., Carrillo, C., Galiana, M.E., García, P. y Trescastro, E.M. (2013). Género y profesión en la evolución histórica de la Enfermería Comunitaria en España. Enfermería Clínica, 23(6), 284-289.

- Camaño, R. (2008). Proceso de profesionalización: evolución de la denominación de enfermería. Híades, 10(2), 903-920.

- Del Puerto, I. (2011). Los cuidados de enfermería durante las guerras coloniales españolas (1895-1926). Repercusiones de ambos conflictos en la provincia de Toledo. Tesis Doctoral. Departamento de Historia. Facultad de Letras. Toledo: Universidad de Castilla La Mancha.

- Domínguez, C. (1986). Los cuidados y la profesión enfermera en España. Madrid: Pirámide.

- Feria, D.J., León, R., Macías, A.C., Barquero, A. Marquínez, M.A. y Cárdenas, M.D. (2004). 
Evolución histórica de los cuidados desde el siglo XIX hasta nuestros días. Híades, 9, 425-451.

- Herrera, F. (1996). La enseñanza de las Damas Enfermeras de la Cruz Roja (1917-1920). Híades, 3, 197-209.

- Herrera, F. (2000). Un capítulo de la enfermería: la cirugía menor en la España del siglo XIX. Cultura de los Cuidados, 7(8), 18-26.

- Ortiz, T. (2005). Profesiones sanitarias. En I. Morant, G. Gómez-Ferrer, D. Barrancos y A. Lavrin, Historia de las mujeres en España y América Latina. Del siglo XIX a los umbrales del XX, 3 (pp. 523-
543). Madrid: Cátedra.

- Parrilla, J. y García, C. (2004). Análisis de la Enfermería en España desde finales del siglo XIX hasta su integración en la Universidad. Híades, 9, 407-424.

- Santo Tomás, M. (1997). Historia de la Enfermería. En C. Fernández, M. Garrido, M. Santo Tomás, M.D. Serrano y R.M. Fuentes, Enfermería Fundamental (pp. 3-131). Barcelona: Masson. 